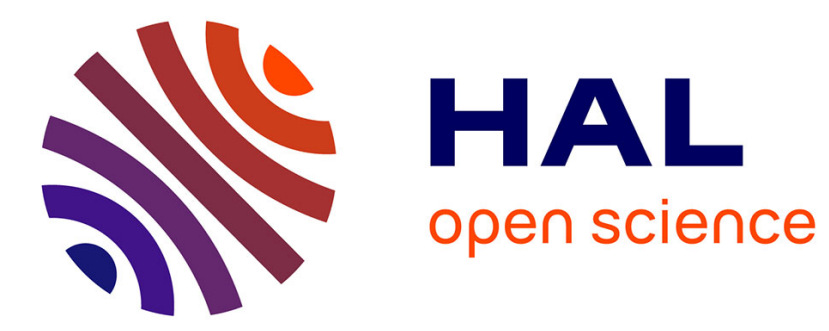

\title{
L'enrichissement des surintendants Bullion et Bouthillier ou le détournement des fonds publics sous Louis XIII Yves Le Guillou
}

\section{To cite this version:}

Yves Le Guillou. L'enrichissement des surintendants Bullion et Bouthillier ou le détournement des fonds publics sous Louis XIII. Dix-septième siècle, 2001, 211 (2), p. 195-213. 10.3917/dss.012.0195 . hal-01778897

\section{HAL Id: hal-01778897 \\ https://hal.science/hal-01778897}

Submitted on 3 May 2018

HAL is a multi-disciplinary open access archive for the deposit and dissemination of scientific research documents, whether they are published or not. The documents may come from teaching and research institutions in France or abroad, or from public or private research centers.
L'archive ouverte pluridisciplinaire HAL, est destinée au dépôt et à la diffusion de documents scientifiques de niveau recherche, publiés ou non, émanant des établissements d'enseignement et de recherche français ou étrangers, des laboratoires publics ou privés. 


\section{L'ENRICHISSEMENT DES SURINTENDANTS BULLION ET BOUTHILLIER OU LE DÉTOURNEMENT DES FONDS PUBLICS SOUS LOUIS XIII}

Yves Le Guillou

Presses Universitaires de France | « Dix-septième siècle »

2001/2 n² 211 | pages 195 à 213

ISSN 0012-4273

ISBN 9782130518839

Article disponible en ligne à l'adresse :

https://www.cairn.info/revue-dix-septieme-siecle-2001-2-page-195.htm

\section{Pour citer cet article :}

Yves Le Guillou, «L'enrichissement des surintendants Bullion et Bouthillier ou le détournement des fonds publics sous Louis XIII ", Dix-septième siècle 2001/2 (n 211), p. 195-213.

DOI 10.3917/dss.012.0195

Distribution électronique Cairn.info pour Presses Universitaires de France.

(C) Presses Universitaires de France. Tous droits réservés pour tous pays.

La reproduction ou représentation de cet article, notamment par photocopie, n'est autorisée que dans les limites des conditions générales d'utilisation du site ou, le cas échéant, des conditions générales de la licence souscrite par votre établissement. Toute autre reproduction ou représentation, en tout ou partie, sous quelque forme et de quelque manière que ce soit, est interdite sauf accord préalable et écrit de l'éditeur, en dehors des cas prévus par la législation en vigueur en France. Il est précisé que son stockage dans une base de données est également interdit. 


\section{L'enrichissement des surintendants Bullion et Bouthillier ou le détournement des fonds publics sous Louis XIII}

\section{INTRODUCTION}

La richesse de certains surintendants des finances a fait l'objet d'études souvent très précises, comme l'article consacré à la fortune d'Abel Servien par Daniel Dessert $^{1}$ ou l'ouvrage sur Fouquet ${ }^{2}$ du même auteur, ou encore celui sur Pomponne de Bellièvre par Olivier Poncet ${ }^{3}$. Mais alors que la surintendance des finances est parfois collégiale ${ }^{4}$, il n'est jusqu'ici jamais arrivé que l'on compare l'évolution des fortunes de deux surintendants exerçant en même temps la même fonction. C'est ce que le présent article se propose de faire à travers les cas des surintendants Claude de Bullion et Claude Bouthillier grâce, d'une part, aux travaux de Jean-Pierre Labatut sur Bullion, publiés dans la revue XVII siècle en $1963^{5}$ et, d'autre part, aux recherches que j'ai menées sur Bouthillier'.

Jean-Pierre Labatut fonde ses recherches sur l'inventaire après décès de Bullion, dressé par le notaire Charles Richer en 1641 et conservé au minutier central des

1. Daniel Dessert, «Fortune politique et politique de la fortune: à propos de la succession du surintendant Abel Servien ", La France d'Ancien Régime, éd. par Jean Jacquart, François Lebrun, Alain Croix, Toulouse, 1984, p. 207-214.

2. Daniel Dessert, Fouquet, Paris, Fayard, 1989.

3. Olivier Poncet, Pomponne de Bellièrre (1529-1607). Un homme d'État au temps des guerres de religion, Paris, École des chartes, 1999.

4. Champigny et Marillac entre 1624 et 1626 ; Bailleul et le comte d'Avaux entre 1643 et 1647 ; Particelli d'Hemery et le même comte d'Avaux entre 1649 et 1650 ; Servien et Fouquet entre 1653 et 1659.

5. Tous les détails sur la fortune de Bullion mentionnés dans le présent article sont tirés de JeanPierre Labatut, «Aspects de la fortune de Bullion », XVIT siècle, 1963, n 60, p. 11-39. Cette référence restera constamment implicite et ne sera plus rappelée en note.

6. Yves Le Guillou, Les Bouthillier, de l'avocat au surintendant (ca 1540-1652). Histoire d'une ascension sociale et formation d'une fortune, thèse d'École des chartes, 1997. 
notaires parisiens ${ }^{7}$. L'étude de la fortune de Claude Bouthillier s'appuie également, en grande partie, sur le minutier central des notaires parisiens et notamment sur les études LXXXVI et CV, avec la collecte de quelque 400 à 500 minutes. Mais le minutier central n'est pas le seul fonds conservant des archives sur Bouthillier. Le fonds de Saxe (série $\mathrm{E}^{*}$ ) des Archives départementales de l'Aube en recèle un bon nombre, et le Répertoire des titres et papiers composant les archives de M. Le marquis de Chavigny (appelé ici Répertoire Chavigny), conservé chez M. le marquis de La Rochelambert, a livré de précieuses informations.

Alors que la fortune du surintendant Bouthillier peut être suivie pas à pas et dans tous les domaines d'investissement, celle du surintendant Bullion n'apparaît avec suffisamment de précision que dans les domaines foncier et immobilier. C'est pourquoi la comparaison qui suit ne tient compte que de la fortune foncière et immobilière des deux personnages. Avant d'aborder celle-ci, il convient de camper les deux surintendants dans leur milieu social et familial.

CARRIÈRE, POSITION SOCIALE ET MARIAGE

\section{Claude Bouthillier}

Claude Bouthillier, fils de Denis Bouthillier, avocat au parlement de Paris, et de Claude de Macheco, est baptisé en l'église Saint-Côme, à Paris, le 4 février $1581^{8}$. Après des débuts comme avocat au parlement de Paris ${ }^{9}$, il devient conseiller au même parlement en $1613^{10}$, puis, grâce à Richelieu, obtient, en 1619 , des lettres de provision pour la charge de secrétaire des commandements de la reine mère ainsi qu'un brevet de conseiller d'État ${ }^{11}$.

En 1628, le roi choisit Bouthillier pour succéder à Nicolas Potier d'Ocquère comme secrétaire d'État ${ }^{12}$. Il lui attribue, l'année suivante, le département des Affaires étrangères ${ }^{13}$ avant de le nommer, en 1632, surintendant des finances en même temps que Bullion. Bouthillier est également nommé intendant de la maison du roi $^{14}$ et surintendant de la maison de Navarre ${ }^{15}$. L'office de secrétaire d'État qu'occupait Bouthillier jusque-là est transmis à son fils Léon, comte de Chavigny. Par un brevet du 18 mars 1632, il obtient pour lui la survivance de cet office ${ }^{16}$. Cependant,

7. MC, ét. LI, 259, 9 janvier 1641.

8. Répertoire Chavigny, fol. $42 \mathrm{v}$.

9. Sa réception date du 12 novembre 1602 (Bibliothèque de l'Ordre des avocats, ms. 359, p. 249).

10. AN, X1a 1858, fol. 157.

11. Répertoire Chavigny, art. 215, fol. 20.

12. Antoine Fauvelet du Toc, Histoire des secrétaires d'État contenant l'origine, le progrès et l'établissement de leurs charges, avec les éloges [...] et généalogies de tous ceux qui les ont possédées jusqu'à présent, Paris, 1668, p. 55-56.

13. BnF, ms. fr. 3673, fol. 79, Louis XIII à Philippe de Béthune, 20 juin 1629.

14. Répertoire Chavigny, art. 208, fol. 19 v.

15. Ibid., art. 163 , fol. $15 \mathrm{v}$.

16. Ibid., art. 182, fol. 17 . 
comme il le déclare lui-même, jamais il n'abandonnera sa fonction de secrétaire d'État:

J'ay, soussigné, reconnu et confessé que le roy m'ayant fait l'honneur de m'appeler à la charge de surintendant de ses finances avec $\mathrm{M}$. de Bullion, j'ay le même jour déclaré à mon fils, ainsy que je déclare par ce présent écrit, que je luy laissois, comme de fait je luy laisse, toute la fonction de ma charge de secrétaire d'État et les apointemens entiers d'icelle, moyennant quoy mon fils me payera la somme de cent cinquante mil livres, de laquelle nous avons convenu ensemble, lorsque le roy eust agréable de luy accorder ma survivance, et, partant lad. charge de secrétaire d'État appartiendra doresnavant du tout à mon fils pour icelle charge ou les deniers qui en pourront provenir, cy après estre propres à luy et à ses enfans, et néantmoins, ainsy qu'il a plust à Sa Majesté, je demeure toujours pourveu d'icelle, retenant la plume pour pouvoir quelquesfois signer aux affaires qui n'ont point d'incompatibilité avec la surintendance des finances, à condition que cy par cy après je revenois à en reprendre l'exercice et les apointements et autres choses susd., je rendrois en ce cas lad. somme de cent cinquante mil livres à mon fils, lequel demeurera au premier terme de la survivance que le roy luy a donnée en ma faveur. ${ }^{17}$

Chavigny est officiellement investi de la charge de secrétaire d'État, il en touche les appointements, mais son père se réserve le droit d'intervenir quand bon lui semble. C'est ce que l'on constate effectivement à la lecture des dépêches envoyées aux ambassadeurs.

Claude Bouthillier a aussi un rôle dans les questions maritimes puisqu'il est conseiller au conseil de la marine à partir de $1632^{18}$.

De 1640 à 1643, Bouthillier administre seul les finances du royaume. Mais après la mort du roi, sa disgrâce est rapide. Louis XIII, par son «testament», exprimait la volonté de garder l'équipe ministérielle de Richelieu. Le roi espérait que la forme du gouvernement qu'il établissait durerait jusqu'en 1652, date à laquelle Louis XIV aurait 14 ans. Il voulait que la Régence continuât la politique entreprise jusqu'ici, notamment la politique extérieure. En plus d'Anne d'Autriche, qui serait devenue régente, et de Gaston d'Orléans, qui serait devenu lieutenant général du royaume, le conseil de régence aurait compris Condé, Mazarin, Séguier, et les deux Bouthillier : Claude, comme surintendant des finances, et Léon, comme secrétaire d'État des affaires étrangères ${ }^{19}$. La reine Anne n'ayant jamais apprécié les créatures de Richelieu, elle fait casser le testament de Louis XIII le 18 mai 1643. Bouthillier est démis de ses fonctions en juin $1643^{20}$ et remplacé par Nicolas de Bailleul et Claude de Mesmes. Pourtant, à la fin du même mois, il est nommé ministre d'État ${ }^{21}$. Mais cette

17. ADA, E* 3061, partage des biens de Léon Bouthillier, novembre 1653 - janvier 1654, lettre du 6 août 1632 .

18. BnF, ms. fr. 6409, fol. 100 .

19. Richard Bonney, The King's debts: finance and politics in France, 1589-1661, Oxford, 1981, p. 190-191.

20. Mathieu Molé, Mémoires, pub. par Aimé Champollion-Figeac, Paris, 1855, t. III, p. 68

21. BnF, Pièces originales $479, \mathrm{n}^{\circ} 169$. 
marque honorifique ne doit pas cacher sa totale disgrâce. Il meurt le 13 mars 1652 à l'âge de 71 ans.

Claude Bouthillier épouse, en août 1606, Marie de Bragelongne ${ }^{22}$, fille de Léon de Bragelongne, conseiller au Parlement de Paris, et de Guyonne de La Grange. Leur contrat de mariage a pu être retrouvé ${ }^{23}$. La dot de la future se compose de deux maisons situées à Garges ${ }^{24}$ et contenant 148 arpents de terres, auxquelles Léon de Bragelongne ajoute la somme de 12000 livres. D'après l'état des biens de 1674, les terres données en dot à Marie de Bragelongne valent alors environ 14000 livres. Le montant total de la dot atteindrait donc 26000 livres. Quant à Denis Bouthillier et sa femme, ils donnent aux futurs époux la terre de Foulletourte ${ }^{25}$ ainsi que 20000 livres à prendre sur leurs biens meubles et immeubles.

\section{Claude de Bullion}

Claude de Bullion, baptisé à Paris, en l'église Saint-André-des-Arts le 13 octobre 1569, est le deuxième fils de Jean de Bullion, conseiller au parlement de Paris et de Charlotte de Lamoignon. Reçu comme avocat au parlement de Paris en 1594, il acquiert, dès 1595, une charge de conseiller au même parlement. En 1605, il devient maître des requêtes et, en 1606, conseiller d'État ainsi que président au parlement de Grenoble. Il est nommé surintendant des finances avec Claude Bouthillier, en 1632, et le roi crée à son profit une charge de premier président au parlement de Paris, où il est reçu en 1636.

Il épouse, en 1612, Angélique Faure, fille de Guichart Faure, riche diplomate, et de Madeleine Brulart, sœur de Nicolas Brulart de Sillery, chancelier de France. La dot s'élève à 75000 livres.

\section{LES HÉRITAGES FAMILIAUX}

\section{L’héritage familial de Bullion}

Le grand-père de Bullion, Claude I $^{\text {er }}$ de Bullion, faisait partie de la bonne bourgeoisie de Mâcon, comme important marchand en gros. Son père, Jean de Bullion, secrétaire du roi, entre, par son mariage avec Charlotte de Lamoignon, dans une excellente famille de robe de la capitale : le père de Charlotte, Charles de Lamoignon, est maître des requêtes de l'hôtel. Jean de Bullion acquiert très vite un office de conseiller au parlement avant de devenir lui-même maître des requêtes, en 1574.

22. C'est l'orthographe «Bragelongne» qui a été retenue, bien que Marie signe toujours «Brageloigne », comme d'ailleurs tous ses parents sur le contrat de mariage. Ce nom est orthographié de manière très diverse selon les historiens : Bragelonne, Bragelongue... «Bragelongne» est la version usitée qui nous a semblé la plus proche de ce que nous avons rencontré.

23. MC, ét. XXIX, 116, 29 août 1606.

24. Val-d'Oise, arr. Montmorency, cant. Garges-les-Gonesse.

25. Cf. infra. 
Alors que la succession de Denis Bouthillier, père de Claude Bouthillier, est connue, il manque l'acte de partage des biens de Jean de Bullion pour la raison qu'il fut passé sous seing privé. Cependant, Jean-Pierre Labatut a pu reconstituer l'héritage qui échoit à Claude de Bullion en 1594: une maison rue du Battoir d'une valeur de 24000 livres, 250 livres de rente annuelle, des droits sur les gabelles du sel du Dauphiné. En outre, son grand-père, Claude Vincent, lui donne la somme de 4000 écus.

\section{L'héritage familial de Bouthillier'}

La famille Bouthillier faisait partie des bonnes familles d'Angoulême. Sébastien Bouthillier, le grand-père du surintendant, était avocat au présidial d'Angoumois et échevin d'Angoulême. Denis Bouthillier, le père du surintendant, s'installe à Paris en 1571 et devient avocat au Grand Conseil avant d'être reçu comme avocat au parlement en 1585. C'est un des principaux avocats de son temps. Il épouse, en 1576, Claude de Macheco, fille d'André de Macheco, auditeur en la chambre des comptes de Dijon, et de Françoise Barjot. Les frères de Françoise Barjot, Claude et Philbert, se sont succédé à la présidence du Grand Conseil.

L'avocat Denis Bouthillier s'enrichit beaucoup durant sa carrière et il laisse à sa mort, en 1621, quelque 220000 livres, dont à peu près 100000 échoient à Claude Bouthillier: 45000 livres provenant de la vente de la seigneurie de Foulletourte, deux maisons à Paris d'une valeur totale de 48000 livres, ainsi que 4000 livres de vaisselle et meubles, et 3000 livres en principal d'une rente.

\section{ÉVOLUTION COMPARATIVE DES FORTUNES FONCIÈRES \\ DE BULLION ET DE BOUTHILLIER}

La fortune de Bullion n'a, jusqu'ici, pas fait l'objet d'une étude aussi précise que celle de Claude Bouthillier. Cependant, l'inventaire après décès dressé à la mort de Bullion permet de se faire une assez bonne idée de ce que furent les acquisitions foncières et immobilières tout au long de sa vie. Bullion acquiert ses domaines dans un rayon de $100 \mathrm{~km}$ au sud de Paris, et notamment la seigneurie de Boulon qu'il fait renommer Bullion par lettres patentes. Le cumul de ces acquisitions peut se résumer par le graphique 1. Elle atteint près de quatre millions de livres en 1640.

Quant à Claude Bouthillier, son domaine principal se constitue en Champagne, autour de la seigneurie des Caves, dont hérite sa femme en $1612^{27}$. Ces acquisitions se font essentiellement de 1628 à 1641, dont plus de $80 \%$ avant $1634^{28}$. À partir

26. Yves Le Guillou, «L'enrichissement de l'avocat Denis Bouthillier (1540-1621) : radiographie d'une fortune », Revue de la société internationale d'bistoire de la profession d'avocat, $\mathrm{n}^{\circ}$ 9, 1997, p. 3-33.

27. Yves Le Guillou, «Un surintendant des finances en Champagne: Claude Bouthillier», La Vie en Champagne, no 11, 1997, p. 5-15.

28. Le tableau 1 permet de suivre l'évolution des investissements de Bouthillier en Champagne durant cette période. 
de 1633, Bouthillier porte ses regards vers la Touraine et achète la châtellenie de Chavigny, près de Chinon, qu'il agrandit rapidement ${ }^{29}$. Enfin, après 1640, Bouthillier déplace ses investissements fonciers vers l'Ile-de-France, au bailliage de Sens ${ }^{30}$.

Le découpage chronologique 1606-1627, 1628-1644 et 1645-1652, qui correspond aux trois périodes d'investissements fonciers par Bouthillier, épouse, presque exactement, la chronologie de sa carrière : 1602-1628 (Bouthillier avocat et conseiller au Parlement de Paris), 1628-1643 (Bouthillier secrétaire d'État et surintendant des finances), 1643-1652 (disgrâce et mort de Bouthillier). Le graphique 2, qui résume l'évolution des acquisitions foncières de Bouthillier au cours de sa vie, donne une bonne image du profit qu'il retire de l'exercice du pouvoir. La fortune foncière du surintendant n'atteint pas 1,2 million de livres en 1652 .

Bien que Bullion, par ses origines et son mariage, appartienne à un milieu social un peu plus prestigieux que Bouthillier et bien que Bouthillier fasse, pour sa part, un plus gros héritage, les fortunes respectives des deux surintendants, au début de leur carrière, sans être équivalentes, suivent une évolution comparable : l'enrichissement des deux ministres date précisément de leur passage à la tête des finances du royaume. Cependant, la fortune foncière de Bouthillier s'accroît beaucoup moins vite que celle de Bullion sur la même période ${ }^{31}$. Entre 1632 et 1634, sa croissance est 1,4 fois moins rapide. Entre 1634 et 1639, elle est 6 fois moins rapide, et après 1639, elle reste 3,4 fois moins rapide. Alors que l'évolution de leurs fortunes foncières est comparable entre 1601-1606 et 1627, c'est-à-dire au début de leur carrière, l'exercice conjoint de la surintendance entre 1632 et 1640 ne les fait pas fructifier dans les mêmes proportions. En moyenne, celle de Bullion s'accroît 2,5 fois plus vite que celle de Bouthillier, ce qui est énorme. Reste à déterminer l'origine de cet enrichissement.

Certes, Claude Bouthillier prend grand soin de l'administration de sa fortune. Il tient le plus possible à veiller personnellement à ses affaires, ne concédant délégation à sa femme que s'il est absent ou dans l'impossibilité de se déplacer. Ainsi, la plupart des contrats touchant les terres de Touraine et de Champagne sont-ils signés par Marie de Bragelongne. Quand il ne les signe pas, Bouthillier les ratifie toujours, et, surtout, il en prend soigneusement connaissance, au point de faire au dos de chaque contrat un résumé de sa main, contenant les informations essentielles ${ }^{32}$. Grâce à une gestion très prudente, il lui est possible de réinvestir. Mais il a beau apporter un soin particulier à la gestion de sa fortune, une politique intelligente d'investissements ne suffit pas à expliquer l'enrichissement spectaculaire du surintendant. Il n'a pas pu avoir assez de temps pour disposer d'une masse financière assez forte qui lui permette des achats importants. Ce qu'il touche en gages et appointements ne suffit pas non plus à éclaircir le problème. Le tableau 4 montre ce

29. Cf. tableau 2 et Yves Le Guillou, «Des Bouthillier aux Chavigny : l'implantation de "créatures" de Richelieu en Touraine», Mémoires de la société archéologique de Touraine, t. XLV, 1998, p. 502-528.

30. Cf. tableau 3.

31. La méthode utilisée pour le calcul de ce qui suit est développée dans Yves Le Guillou, «Un outil pour l'exploitation des graphiques en histoire : la régression linéaire », Revue informatique et statistique dans les sciences humaines, 1998, p. 119-123.

32. Presque toutes les pièces trouvées dans les archives de M. du Petit-Thouars, de M. de Soyres et aux archives départementales de l'Aube possèdent ce résumé. 
que perçoit Bouthillier en fonction des charges dont il est pourvu. Le maximum atteint 30000 livres par an entre 1634 et 1642. Le cumul des gages, pensions et appointements au fil des années ne dépasse pas les 650000 livres en 1652, d'après toutes les sources rassemblées. Il faut donc chercher ailleurs la cause de l'enrichissement des surintendants.

\section{L'ORIGINE OCCULTE D’UNE FORTUNE MINISTÉRIELLE}

La courbe d'évolution de la fortune des surintendants, qui varie avec leurs fonctions ministérielles, montre que cette source s'alimente au service du roi. Le gros des revenus tirés de l'exercice du pouvoir ne vient ni des gages ni des appointements ni des pensions, mais il a une ou plusieurs origines dont la caractéristique est de ne pas laisser de trace dans les archives. En raison de leur nature secrète, il est difficile de décrire ces sources de profit; certains indices permettent toutefois de s'en faire quelque idée.

\section{Les dons}

Une seule trace de don a pu être trouvée concernant Claude Bouthillier. En 1624, Marie de Médicis donne à ce dernier un terrain en face de l'hôtel du Luxembourg, dans la vieille rue de Vaugirard ${ }^{33}$. Ce terrain est estimé à 30000 livres $^{34}$. Nous n'avons connaissance de ce don que par les actes établis pour la succession de Marie de Bragelongne en 1673 et 1674 et non par les actes notariés ordinaires, car ce genre d'actes est, le plus souvent, passé sous seing privé. L'absence totale de dons de la part de Richelieu ou Louis XIII à Bouthillier laisse à penser que leurs traces ont disparu avec les archives de la famille. Il est, en effet, impossible d'imaginer que le cardinal n'ait pas fait de dons à un ami aussi proche que Bouthillier, alors qu'il fait à Bullion, tous les ans, le $1^{\text {er }}$ janvier, un don de 100000 livres $^{35}$.

\section{Les détournements de fonds publics}

À côté des dons royaux ou princiers, il est de multiples moyens de s'enrichir de «manière détournée ». Au quotidien, l'emploi peu scrupuleux des finances de l'État se fait par le biais des ordonnances de comptants.

C'est en effet ce que laisse entendre Richelieu dans une lettre à Bullion en 1639:

Je prie [M. de Bullion] de résoudre ce qui s'ensuit. [...] À borner tellement sa fortune au bien qu'il a, qu'il n'en désire à l'avenir aucun accroissement, ains se contenter, en servant le roi en la charge en laquelle il est, des gages et appointemens d'icelle,

33. MC, ét. LXXV, 167, 4 septembre 1673, art. 1 bis, 26 février 1624.

34. ADA, E* 3060.

35. P. Henri Griffet, Histoire du rìgne de Louis XIII, Paris, 1758, t. III, p. 302. 
selon le pied auquel ils ont esté restraints par le règlement faict par Sa Majesté le 16 juillet 1638. [...] À restablir l'ordre ancien, suivant lequel on ne doit expédier aucun comptant, de quelque nature que ce puisse estre, soit pour raison de dons, affaires secrètes, estrangères ou autres, ou pour les remises des traictés qui se font au conseil, qu'au mesme temps on en retienne un menu, arresté au conseil de trois en trois mois, signé de messieurs le chancelier, les surintendans et tous les intendans; duquel menu sera faict trois copies, l'une pour le trésorier de l'Espargne, la seconde pour messieurs les surintendans, et la troisiesme pour monsieur le chancelier. Le tout selon qu'il se pratiquoit auparavant la mauvaise introduction de brusler le menu desd. comptants. [...] À s'appliquer aussy soigneusement à la réformation des finances et au soulagement du peuple qu'il s'est attaché à ses affaires particulières avant qu'il fust chargé des publiques. Ce dont il est prié, non seulement à raison des intérests publics, mais en outre afin qu'il soit un jour aussy opulent au ciel qu'il l'est en terre; ce qui est le plus avantageux souhait que luy puisse faire une personne qui l'ayme comme moy. ${ }^{36}$

Le comptant ou ordonnance de comptant est un ordre de paiement sur papier signé du roi ou du surintendant des finances, qui exerce, par délégation, la fonction royale d'ordonnateur principal des dépenses de l'État. L'ordonnance de comptant est ensuite adressée au comptable, par exemple le trésorier de l'Épargne. Une fois le paiement effectué, l'ordonnance est détruite; on délivre alors au comptable une lettre patente scellée du grand sceau portant quittance qui lui sert de décharge devant la chambre des comptes. Cette lettre patente porte le nom d'acquit patent.

Jusqu'en 1634, le conseil des finances garde le menu de tous les paiements (menus de comptants). Quand Claude Cornuel devient intendant des finances, en 1634, il commence par brûler ces registres détaillés, certainement avec l'approbation de Bullion. C'est à cette époque que Cornuel et Bullion bâtissent leur fortune $^{37}$, celle de Cornuel s'élevant à 1,8 million de livres à sa mort ${ }^{38}$. Après 1639 , on cesse de brûler les menus ${ }^{39}$, comme l'a ordonné Richelieu.

Les comptants sont utilisés pour les dépenses secrètes de l'État. Théoriquement, les paiements de cette sorte concernent les affaires qui pourraient mettre le royaume en danger. Dans les faits, on utilise ce mode de paiement pour des raisons politiques et pour des emplois qu'on veut garder secrets. Les dépenses de la Couronne, en effet, sont apurées par le conseil d'État et des finances, et envoyées à la chambre des comptes où elles sont vérifiées une seconde fois. Il semble que cette seconde vérification, très critiquée des contemporains comme étant trop formelle, ait été en général faite sérieusement. Cependant, alors que les autres chapitres sont contrôlés rigoureusement, les dépenses secrètes ne le sont pas. C'est la raison pour laquelle les surintendants utilisent abondamment ce genre de paiement ${ }^{40}$. Colbert, quelques

36. Denis Avenel, Lettres, instructions diplomatiques et papiers d'État du cardinal de Richelieu, Paris, t. VI, 1867, p. 271.

37. Richard Bonney, op. cit., p. 181.

38. Julian Dent, Crisis in finance: Crown, financiers and society in 17th century in France, New Abbot, 1973, p. 167.

39. Richard Bonney, op. cit., p. 183.

40. Richard Bonney, «The secret expenses of Richelieu and Mazarin 1624-1661 », English historical review, XCI, 1976, p. 826. 
années plus tard, voit dans les comptants la source des détournements financiers. «Sous couvert de ce secret, dit-il, se cachent tous les abus et toutes les malversations qui se commettent dans les finances $»^{41}$.

L'évolution des recettes et des dépenses de l'État se caractérise, dans la première moitié du XVII siècle, par un formidable accroissement, et les ponctions opérées par la monarchie à la même époque sont gigantesques. Vers 1580, les recettes s'élèvent à près de 14500000 livres; en 1635, elles sont de $208000000^{42}$. La hausse est particulièrement prodigieuse entre 1631 et 1635 . À ce moment-là, les recettes ne font pas moins que sextupler ${ }^{43}$.

Françoise Bayard divise les dépenses de la monarchie en trois catégories: les dépenses de la cour, les dépenses pour la politique extérieure, et les comptants. Alors que les dépenses de la cour augmentent raisonnablement, les coûts de la politique étrangère enflent particulièrement et arrivent à un premier maximum en 1635 , et à un second en 1643, ne commençant à diminuer qu'à partir de $1644^{44}$. Quant aux comptants, ils grossissent allègrement à partir de 1629, avec un sommet, en 1635, de plus de 155300000 livres. Les dépenses politiques leur sont inférieures à partir de 1631, et parfois de bien plus de la moitié. Ainsi, en 1634, les comptants atteignent-ils plus de 84 millions de livres alors que les dépenses de politique extérieure ne montent pas à 27 millions. En 1635, avec 43450000 livres, les dépenses de politique extérieure sont plus de 3,5 fois inférieures aux comptants ${ }^{45}$.

Dès 1614 , les États généraux tentent de s'attaquer à tous ces abus. Ils proposent que les ministres présentent à la chambre des comptes une déclaration complète de leurs biens. Le tiers état réclame la suppression du paiement des dépenses secrètes par les comptants. Les pensions et dons de la régente sont jugés excessifs. Les noms des vrais bénéficiaires de ces derniers devraient être fixés par lettres patentes. Les dons supérieurs à 3000 livres devraient être vérifiés et enregistrés par la chambre des comptes ${ }^{46}$.

De son côté, le roi essaie régulièrement de porter remède à la situation. L'arrêt du Conseil d'État du 13 septembre 1628 ordonne qu'un registre soit tenu de tous les titres concernant les affaires du roi et que ce registre soit conservé au contrôle général des finances. Ce registre renfermerait tant les traités, marchés, remboursements, engagements des domaines que

les ordonnances de comptants expédiées pour acquiter les despences de la guerre et autres despences publiques desquelles l'on n'a peu tirer les acquitz en forme pour la descharge de Sa Mté [art. 13], comme aussi les ordonnances de comptans qui

41. P. Clément, Lettres, instructions et mémoires de Colbert, t. II, 1870, p. 28-29.

42. Françoise Bayard, Le Monde des financiers au XVII' siècle, Paris, 1988, p. 29-30.

43. Ibid., p. 31.

44. Ibid., p. 34

45. Ibid., p. 33.

46. Richard Bonney, The King's debts [...], op. cit., p. 82. 
s'expédient pour acquiter les debtes ou pensions deues aux étrangers dont le payement se faict ouvertement et autres semblables despences qui pouvaient estre demandées deux foys s'il n'en estait gardé mémoire [art. 14] ${ }^{47}$;

autrement dit, concernant les ordonnances de comptants, ce registre servirait à garder trace de documents que l'on a l'habitude de détruire. Sans doute ces registres (ou menus) ont-ils été tenus jusqu'à ce que Bullion les détruise. D'ailleurs, le 16 juillet 1638 , le roi réitère sa volonté de contrôler ses finances en promulgant un règlement ${ }^{48}$ qui devrait « estre à l'advenir inviolablement observé en ses finances ». C'est de ce règlement que parle Richelieu dans sa lettre à Bullion. Mais ce règlement, qui traite essentiellement de la question des comptants, ne sera pas plus appliqué par Bullion.

Comment Richelieu a-t-il eu vent des pratiques de Bullion? Sans doute par Bouthillier. C'est en tout cas ce que laisse penser une formule énigmatique du cardinal à la fin d'une lettre à Bouthillier : «Il y a beaucoup de choses à démesler avec M. de Bullion dont vous sçavés seul le détail. » Orest Ranum y a vu l'expression de la perplexité de Richelieu devant l'écheveau des problèmes financiers ${ }^{49}$. Les deux lettres étant datées du même jour, il s'agit sans doute des pratiques de Bullion que Richelieu demande à Bouthillier d'observer de plus près. Il faut de plus remarquer qu'à cette époque, Bullion effectue rapidement ses paiements quand il achète des maisons ou des terres. Cette façon de faire peut être assimilée à une sorte de "blanchiment» d'un argent mal acquis.

\section{CONCLUSION}

Bullion et Bouthillier se sont considérablement enrichis au cours de la période qu'ils ont passée à la tête des finances du royaume; mais pas dans les mêmes proportions. Est-ce à dire que Bouthillier fut plus scrupuleux que Bullion? C'est ce que pense son neveu Jean de La Barde, qui considère que c'est son intégrité qui a valu à Bouthillier de se faire évincer de la surintendance :

Ipse unus [Claudius Butbillerius] per triennium hunc magistratum gessit, magna cum probitatis atque integritatis fama, quam ipsi denique fraudi fuisse plerique existimavere: nam id obstabat quominus alii per rei publica damna familiari augenda, ut fere sit, operam darent; quamobrem buic summam quasturam abrogatam volebant. ${ }^{50}$

47. Roland Mousnier, «Les règlements du conseil du roi sous Louis XIII », Annuaire-bulletin de la Société de l'bistoire de France, 1946-1947, p. 180.

48. Archives des Affaires étrangères, France, 806, fol. 178

49. Orest Ranum, Les Créatures de Richelieu, secrétaires d'État et surintendants des finances 1635-1642, Paris, 1966, p. 206.

50. Jean de La Barde], Joannis Labardai, Matrolarum ad Sequanam marchionis, regis ad Helvetios et Rhaetos extra ordinem legati, De rebus gallicis historiarum libri decem, ab anno 1643 ad annum 1652, Paris, 1671, p. 6. [Pendant trois ans, seul [Claude Bouthillier], il assuma cette charge avec une réputation d'honnêteté et d'intégrité dont beaucoup pensèrent qu'il l'avait, en fin de compte, usurpée; car il s'opposait à ce que certains s'appliquassent à s'enrichir au détriment de l'État. C'est pourquoi ceux-ci voulaient que la surintendance lui fût retirée]. 
L'étude de la gestion du trésor des ordres du roi parait plus convaincante.

Nommé trésorier des ordres en 1633, Bouthillier se charge d'éponger la dette de 750000 livres laissée par son prédécesseur Thomas Morant. Rien d'étonnant à ce qu'une telle dette ait pu s'accumuler au fil des années : les comptes ne sont vérifiés qu'une fois le trésorier démis de sa charge. Ainsi Pierre Brûlart, sieur de Puysieux, devait-il à Morant 194085 livres 1 sol 2 deniers et 306 écus d'or après la vérification de ses comptes le 17 mars $1622^{51}$. Les vérifications sont plus fréquentes avec Bouthillier, puisqu'elles ont lieu en 1643, 1649, 1651 et 1652, après la mort de Bouthillier, car Bouthillier reste trésorier des Ordres jusqu’à sa mort, comme en témoigne le paiement de ses gages pour le quartier de janvier $1652^{52}$. Sans doute la somme énorme que doit Thomas Morant au terme de l'exercice de ses fonctions a-t-elle eu pour effet quelques réformes dans l'administration des finances des ordres du roi. À la fin de l'année 1651, Bouthillier continue toujours de poursuivre les héritiers de son prédécesseur pour le remboursement de 143283 livres 17 sols 2 deniers $^{53}$, alors qu'il a déjà obtenu contre Morant deux arrêts du conseil : l'un le 16 décembre $1633^{54}$ et l'autre le 12 octobre $1649^{55}$. Au contraire de ses prédécesseurs, c'est le trésor royal qui doit de l'argent à Bouthillier après vérification de ses comptes; en 1659, les 723 dernières livres sont payées à Marie de Bragelongne ${ }^{56}$.

La gestion du trésor des ordres du roi par Claude Bouthillier laisse à penser que ce dernier a géré le trésor royal avec la même loyauté. Il resterait maintenant à étudier en détail l'activité ministérielle des deux surintendants pour mettre à jour, exemples à l'appui, les procédés frauduleux employés par Bullion.

Yves Le Guillou, archiviste paléographe, conservateur des bibliothèques, Bibliothèque universitaire, Faculté Jean-Monnet, Sceaux.

51. BnF, Clairambault 1247, fol. $36 \mathrm{v}$.

52. BnF, Clairambault 1248, fol. 114.

53. MC, ét. LXXXVI, 383, 18 décembre 1651.

54. MC, ét. LXXXVI, 377, 4 juillet 1647.

55. Bibliothèque de l'Institut, Godefroy 274, fol. 131, lettre de Claude Bouthillier au [chancelier Séguier], 23 octobre 1649.

56. BnF, Clairambault 1248, fol. 182, quittance du $1^{\text {er }}$ septembre 1659. 


\section{ANNEXES}

TABLEAU 1 - Les investissements fonciers de Claude Bouthillier en Champagne

\begin{tabular}{|c|c|c|}
\hline Date & Valeur & Objet (source) \\
\hline 1616 (13 septembre) & $\begin{array}{l}300+ \\
1 / 2 \text { maison }\end{array}$ & $\begin{array}{l}\text { 4,5 a à Pont (MC, ét. LXXV, 167, } \\
4 \text { septembre 1673) }\end{array}$ \\
\hline 1618 (23 août) & 1000 & 10 a à Pont (MC, ét. CV, 568, 23 août 1618) \\
\hline 1619 (26 janvier) & 5000 & 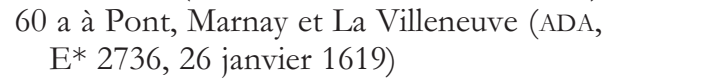 \\
\hline 1625 (23 janvier) & 7000 & $\begin{array}{l}69 \text { a à Pont et Crancey (ADA, E* 2737, } \\
23 \text { janvier 1625) }\end{array}$ \\
\hline 1626 (16 juillet) & 84 & $\begin{array}{l}3,5 \text { q près des Caves (ADA, E* } 2737 \text {, } \\
16 \text { juillet } 1626 \text { ) }\end{array}$ \\
\hline 1627 (26 janvier) & 525 & 7 a à Pont (MC, ét. LXXV, 167, 4 septembre 1673) \\
\hline 1628 (5 mars) & 16000 & fief de Besançon (MC, ét. LXXXVI, 4 février 1628) \\
\hline 1628 (7 août) & 1000 & $\begin{array}{l}\text { une maison et 20,5 a à Marnay (ADA, E* 3009, } \\
7 \text { août 1628) }\end{array}$ \\
\hline 1628 (8 août) & 550 & 13 a de bois à Marnay (ADA, E* 3009, 8 août 1628) \\
\hline 1629 (9 août) & 20 & $\begin{array}{l}\text { petit jardin à Pont (MC, ét. LXXV, 167, } \\
4 \text { septembre } 1673)\end{array}$ \\
\hline 1630 (22 juin) & 24000 & $\begin{array}{l}\text { seigneurie de Foujon (Le Clert, Quelques } \\
\text { seigneuries..., p. } 27 \text { ) }\end{array}$ \\
\hline 1630 (20 novembre) & 150 & $\begin{array}{l}\text { une maison et } 1 / 2 \text { a à Marnay (MC, ét. LXXV, } 167 \text {, } \\
4 \text { septembre } 1673 \text { ) }\end{array}$ \\
\hline 1630 (25 novembre) & 100 & 1 a à Marnay (ADA, E* 3009, 25 novembre 1630) \\
\hline 1631 (23 octobre) & 20250 & $\begin{array}{l}\text { seigneurie des Vignes (MC, ét. LXXXVI, 236, } \\
12 \text { novembre 1629) }\end{array}$ \\
\hline 1631 (2 décembre) & 36000 & $\begin{array}{l}\text { seigneurie de Crancey (MC, ét. LXXXVI, 245, } \\
4 \text { février 1633) }\end{array}$ \\
\hline
\end{tabular}




\begin{tabular}{|c|c|c|}
\hline Date & Valeur & Objet (source) \\
\hline 1632 (12 juin) & 900 & 3 a à Pont (MC, ét. LXXV, 167, 4 septembre 1673) \\
\hline 1632 (22 août) & 123489,5 & seigneurie de Pont (ADA, E* 2731, 22 août 1632) \\
\hline 1632 (19 décembre) & 225 & 1,5 a à Marnay (ADA, E* 2896, 19 décembre 1632) \\
\hline 1633 (4 février) & 33650 & $\begin{array}{l}\text { seigneurie de Crancey (MC, ét. LXXXVI, 245, } \\
4 \text { février 1633) }\end{array}$ \\
\hline 1633 (4 avril) & 500 & $\begin{array}{l}\text { une grange à Pont (MC, ét. LXXV, } 167 \text {, } \\
4 \text { septembre 1673) }\end{array}$ \\
\hline 1633 (9 juillet) & 18000 & $\begin{array}{l}\text { seigneurie de Pars (MC, ét. LXXXVI, 247, } \\
9 \text { juillet 1633) }\end{array}$ \\
\hline 1633 (18 novembre) & 63800 & $\begin{array}{l}\text { seigneurie de Nogent (MC, ét. LXXXVI, 247, } \\
18 \text { novembre 1633) }\end{array}$ \\
\hline 1634 (6 mai) & 30 & 0,5 a à Foujon (ADA, E* 2950, 6 mai 1634) \\
\hline 1634 (10 mai) & 100 & $\begin{array}{l}2 \text { a à Foujon (MC, ét. LXXV, 167, } \\
4 \text { septembre 1673) }\end{array}$ \\
\hline 1634 (21 juin) & 51 & $\begin{array}{l}1 \text { a de terre à Foujon (MC, ét. LXXV, 167, } \\
4 \text { septembre 1673) }\end{array}$ \\
\hline 1635 (17 janvier) & 21 & 3 d à Foujon (ADA, E* 2950, 17 janvier 1635) \\
\hline 1635 (18 avril) & 152 & terres à Foujon (ADA, E* 2950, 18 avril 1635) \\
\hline 1635 (17 octobre) & 50 & 2 a à Foujon (ADA, E* 2950, 17 octobre 1635) \\
\hline 1636 (6 avril) & $70 \mathrm{~s}$. & $\begin{array}{l}1,5 \text { q à Foujon (Mesnil) (ADA, E* 2950, } \\
6 \text { avril 1636) }\end{array}$ \\
\hline 1636 (22 avril) & 82,4 & $\begin{array}{l}1 \text { a } 1 \text { q } 5 \text { d } 16 \text { c à Pars (ADA, E*2983, } \\
22 \text { avril 1636) }\end{array}$ \\
\hline 1636 (23 avril) & 40 & 9 d 7 c à Pars (ADA, E* 2983, 23 avril 1636) \\
\hline 1636 (29 avril) & 200 & 5 a à Foujon (ADA, E* 2950, 29 avril 1636) \\
\hline 1636 (5 juin) & 30 & $\begin{array}{l}1 / 2 \text { a de terre au finage de Longperche } \\
\text { (MC, ét. LXXV, 167, } 4 \text { septembre 1673) }\end{array}$ \\
\hline 1636 (20 novembre) & 500 & $\begin{array}{l}\text { petite maison à Pont (MC, ét. LXXV, 167, } \\
4 \text { septembre } 1673 \text { ) }\end{array}$ \\
\hline 1636 (13 décembre) & 500 & 6 q à Pont (MC, ét. LXXV, 167, 4 septembre 1673) \\
\hline 1637 (12 mai) & 387 & 12 a à Pars (ADA, E* 2983, 12 mai 1637) \\
\hline 1637 (30 octobre) & 38 & $\begin{array}{l}\text { 1,5 q à Pont (MC, ét. LXXV, } 167 \text {, } \\
4 \text { septembre } 1673)\end{array}$ \\
\hline 1638 (18 janvier) & 36000 & $\begin{array}{l}\text { héritages à Pont (MC, ét. LXXXVI, 256, } \\
18 \text { janvier 1638) }\end{array}$ \\
\hline 1638 (17 juin) & 900 & 2 a 1 q à Pont (ADA, E* 2740, 17 juin 1638) \\
\hline 1638 (17 juin) & 450 & 5 q à Pont (MC, ét. LXXV, 167, 4 septembre 1673) \\
\hline 1638 (13 juillet) & 150 & $\begin{array}{l}\text { fief de Charmoy (MC, ét. LXXV, 167, } \\
4 \text { septembre 1673) }\end{array}$ \\
\hline 1638 (4 novembre) & 3400 & $\begin{array}{c}7 \text { a } 4 \text { q à Crancey, } 2 \text { maisons à Nogent, } 23 \text { a à } \\
\text { Foujon (ADA, E* 3030, } 4 \text { novembre 1638) }\end{array}$ \\
\hline 1639 (9 juin) & 1500 & $\begin{array}{l}\text { une maison à Pont (MC, ét. LXXV, } 167 \text {, } \\
4 \text { septembre 1673) }\end{array}$ \\
\hline 1639 (14 juin) & 504 & 3 a 1 q à Marnay (ADA, E* 3009, 14 juin 1639) \\
\hline 1639 (2 septembre) & 48 & 2 bdc à Pont (ADA, E* 2740, 2 septembre 1639) \\
\hline 1639 (2 septembre) & 700 & 2 jardins à Pont (ibid.) \\
\hline 1639 (3 septembre) & 24 & 1 bdc à Pont (ADA, E* 2740, 3 septembre 1639) \\
\hline
\end{tabular}




\begin{tabular}{|c|c|c|}
\hline Date & Valeur & Objet (source) \\
\hline 1639 (10 septembre) & 72 & 3 bdc à Pont (ADA, E* 2740, 10 septembre 1639) \\
\hline 1639 (10 septembre) & 48 & 2 bdc à Pont (ibid.) \\
\hline 1639 (12 septembre) & 60 & $\begin{array}{l}1 / 2 \text { maison à Foujon (ADA, E* } 2950, \\
12 \text { septembre } 1639)\end{array}$ \\
\hline 1639 (13 septembre) & 24 & 1 bdc à Pont (ADA, E* 2740, 13 septembre 1639) \\
\hline 1639 (13 septembre) & 48 & 2 bdc à Pont (ibid.) \\
\hline 1639 (13 septembre) & 48 & 2 bdc à Pont (ibid.) \\
\hline 1639 (15 septembre) & 24 & 1 bdc à Pont (ADA, E* 2740, 15 septembre 1639) \\
\hline 1639 (15 septembre) & 48 & 2 bdc à Pont (ibid.) \\
\hline 1639 (18 septembre) & 30 & $\begin{array}{l}5 / 4 \text { chènevière à Pont (ADA, E* } 2740, \\
18 \text { septembre } 1639)\end{array}$ \\
\hline 1639 (3 octobre) & 48 & 2 bdc à Pont (ADA, E* 2740, 3 octobre 1639) \\
\hline 1639 (9 octobre) & 12 & 0,5 bdc à Pont (ADA, E* 2740, 9 octobre 1639) \\
\hline 1639 (3 novembre) & 30 & $\begin{array}{l}5 / 4 \text { chènevière à Pont }\left(\mathrm{ADA}, \mathrm{E}^{*} 2740,\right. \\
3 \text { novembre } 1639)\end{array}$ \\
\hline 1639 (22 novembre) & 40 & $\begin{array}{l}5 \text { q de terres au Mesnil (MC, ét. LXXV, 167, } \\
4 \text { septembre 1673) }\end{array}$ \\
\hline 1639 (24 décembre) & 54 & $\begin{array}{l}\text { 2,25 bdc à Pont (ADA, E* 2740, } \\
24 \text { décembre 1639) }\end{array}$ \\
\hline 1640 (7 février) & 16000 & 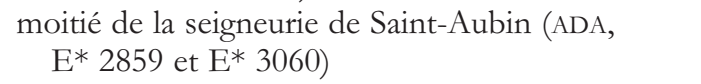 \\
\hline 1640 (8 février) & 36 & 1,5 a à Pont (ADA, E* 2740, 8 février 1640) \\
\hline 1640 (10 mai) & 30 & $\begin{array}{l}20 \text { c de prés à Pont (MC, ét. LXXV, 167, } \\
4 \text { septembre 1673) }\end{array}$ \\
\hline 1640 (9 juin) & 1500 & 1 maison à Pont (ADA, E* 2742, 9 juin 1640) \\
\hline 1640 (7 septembre) & 2400 & $\begin{array}{l}1 / 4 \text { de la seigneurie de la Chapelle-Godefroy } \\
\text { (MC, ét. LXXXVI, 266, } 7 \text { septembre 1640) }\end{array}$ \\
\hline 1641 (9 avril) & 4500 & $\begin{array}{l}1 / 2 \text { de la seigneurie de la Chapelle-Godefroy } \\
\text { (MC, ét. LXXXVI, 269, } 9 \text { avril 1641) }\end{array}$ \\
\hline 1641 (3 juillet) & 1200 & $\begin{array}{l}4 \text { a } 1 \text { q de prés à Marnay (MC, ét. LXXV, 167, } \\
4 \text { septembre 1673) }\end{array}$ \\
\hline 1641 (26 août) & 1400 & $\begin{array}{l}19 \text { a } 1 \text { q 6,5 perches près Nogent } \\
\text { (MC, ét. LXXXVI, 270, } 26 \text { août 1641) }\end{array}$ \\
\hline 1643 (15 avril) & 28,2 & $\begin{array}{l}1 / 2 \text { a } 1 / 2 \text { q au Mesnil (MC, ét. LXXV, 167, } \\
4 \text { septembre } 1673 \text { ) }\end{array}$ \\
\hline 1643 (19 octobre) & 1000 & $\begin{array}{l}4 \text { a de prés à Marnay (MC, ét. LXXV, 167, } \\
4 \text { septembre 1673) }\end{array}$ \\
\hline 1644 (21 avril) & 171 & 4 a 3 q à Pars (ADA, E* 2983, 21 avril 1644) \\
\hline 1644 (21 avril) & 36 & 1 a à Pars (ibid.) \\
\hline 1644 (16 mai) & 48 & 2 a à Pont (MC, ét. LXXV, 167, 4 septembre 1673) \\
\hline 1644 (26 mai) & 40 & 0,5 a à Pont (ADA, E* 2742, 26 mai 1644) \\
\hline 1644 (29 juin) & 1408 & 5,5 a à Pont (ADA, E* 2742, 29 juin 1644) \\
\hline 1644 (29 juin) & 3000 & $\begin{array}{l}33 \text { a } 1 \text { q de bois à Marnay (ADA, E* 3009, } \\
29 \text { juin 1644) }\end{array}$ \\
\hline 1644 (11 décembre) & 75 & $\begin{array}{l}4 \text { bdc } 0,5 \text { q à Foujon (ADA, E* 2950, } \\
11 \text { décembre 1644) }\end{array}$ \\
\hline 1645 (11 avril) & 400 & 1 jardin à Pont (ADA, E* 2742, 11 avril 1645) \\
\hline
\end{tabular}




\begin{tabular}{|c|c|c|}
\hline Date & Valeur & Objet (source) \\
\hline 1645 (28 avril) & 225 & 2 a 1 q à Pont (ADA, E* 2742, 28 avril 1645) \\
\hline 1645 (28 avril) & $\begin{array}{c}\text { [95] (100 par } \\
\text { arpent) }\end{array}$ & 91 c à Marnay (ADA, E* 3009, 28 avril 1645) \\
\hline 1645 (5 mai) & 60 & $1 \mathrm{a}$ au parc de Pont (ADA, E* 2742, 5 mai 1645$)$ \\
\hline 1645 (5 juillet) & 1025 & $\begin{array}{l}\text { une maison à la Villeneuve (MC, ét. LXXV, 167, } \\
4 \text { septembre 1673) }\end{array}$ \\
\hline 1646 (28 avril) & $\begin{array}{l}\text { [64] (100 par } \\
\text { arpent) }\end{array}$ & $\begin{array}{l}62 \text { c à Pont (MC, ét. LXXV, 167, } \\
4 \text { septembre } 1673)\end{array}$ \\
\hline 1646 (28 avril) & 225 & $\begin{array}{l}2,25 \text { a à Pont (MC, ét. LXXV, 167, } \\
4 \text { septembre 1673) }\end{array}$ \\
\hline 1649 (12 avril) & 150 & $1 \mathrm{q}$ de jardin à Pont (ADA, E* 2742, 12 avril 1649) \\
\hline 1650 (28 juin) & 5000 & $\begin{array}{l}\text { terres, maison, tronçons de rivière, etc. à Pont } \\
(\text { ADA, E* 3030, } 28 \text { juin } 1650)\end{array}$ \\
\hline 1651 (7 février) & 75 & $\begin{array}{l}1 \text { q de terre à Faverolle (MC, ét. LXXV, 167, } \\
4 \text { septembre 1673) }\end{array}$ \\
\hline
\end{tabular}

TABLEAU 2 - Les investissements fonciers de Claude Bouthillier en Touraine

\begin{tabular}{|c|c|c|}
\hline Date & Valeur & Objet (source) \\
\hline 1634 (20 août) & 123000 & $\begin{array}{l}\text { seigneurie de Chavigny (MC, ét. LXXXVI, 249, } \\
20 \text { août 1634) }\end{array}$ \\
\hline 1635 (20 mars) & 30000 & $\begin{array}{l}\text { seigneurie de Cécigny (MC, ét. LXXXVI, 250, } \\
20 \text { mars 1635) }\end{array}$ \\
\hline 1635 (30 juin) & 900 & maison de La Bourrie (As) \\
\hline 1635 (30 juin) & 9000 & partie de La Bourrie (AS) \\
\hline 1635 (14 novembre) & 550 & 2 a de prés à Chavigny (As) \\
\hline 1635 (15 novembre) & 100 & 10 b de terre à Chavigny (AS) \\
\hline 1635 (19 novembre) & 1100 & 4,5 a de prés à Chavigny (AS) \\
\hline 1637 (23 février) & 30000 & $\begin{array}{l}\text { seigneurie de Villiers-Boivin (MC, ét. LXXXVI, } \\
\text { 254, } 23 \text { février 1637) }\end{array}$ \\
\hline 1637 (27 juin) & 18800 & maison et terres à La Bourie (AS) \\
\hline 1640 (27 janvier) & $\begin{array}{l}1 / 2 \text { des bancs } \\
\text { de Saumur }\end{array}$ & seigneurie de Lerné (AS) \\
\hline 1640 (7 mars) & 15966 & $1 / 2$ des bancs de Saumur (AS) \\
\hline 1640 (28 avril) & 4800 & $\begin{array}{l}\text { fief de Saint-Germain-des-Prés-les-Candes (MC, ét. } \\
\text { LXXXVI, 265, } 28 \text { avril 1640) }\end{array}$ \\
\hline 1642 (20 février) & 54000 & $\begin{array}{l}\text { seigneurie de la Grande-Gaudrée (MC, ét. } \\
\text { LXXXVI, 272, } 20 \text { février 1642) }\end{array}$ \\
\hline 1645 (22 octobre) & 86 & $3 \mathrm{~b}$ de terre à Chavigny (AS) \\
\hline 1645 (4 novembre) & 150 & 1 a à La Rochethibaud (AS) \\
\hline 1646 (15 septembre) & 14500 & seigneurie de Louzaie (AS) \\
\hline 1647 (19 novembre) & 10 & $1 \mathrm{~b}$ de vigne à Chavigny (As) \\
\hline
\end{tabular}


TABLEAU 3 - Les investissements fonciers de Claude Bouthillier en Ile-de-France

\begin{tabular}{|c|c|c|}
\hline Date & Valeur & Objet (source) \\
\hline$[1640]$ & 73000 & seigneurie de Michery (ADA, E* 3060) \\
\hline 1640 (2 avril) & 300 & $\begin{array}{l}\text { une maison à Michery (MC, ét. LXXV, 167, } \\
4 \text { septembre 1673) }\end{array}$ \\
\hline 1640 (14 octobre) & 717 & $\begin{array}{l}1 / 2 \text { a } 4 \text { q } 33 \text { c de vignes à Michery (MC, ét. LXXV, } \\
\text { 167, } 4 \text { septembre 1673) }\end{array}$ \\
\hline 1641 (11 janvier) & 20000 & $\begin{array}{l}\text { partie de la seigneurie de Sergines } \\
\quad(\text { MC, ét. LXXXVI, 268, } 11 \text { janvier 1641) }\end{array}$ \\
\hline 1641 (9 juillet) & 1330 & $\begin{array}{l}\text { une maison à Michery (MC, ét. LXXV, 167, } \\
4 \text { septembre 1673) }\end{array}$ \\
\hline 1641 (13 octobre) & $\begin{array}{l}{[60000] \text { (rente }} \\
\quad \text { de } 3000 \text { l.) }\end{array}$ & $\begin{array}{l}\text { terres de Lespine, Boisépinard et La Mothe } \\
\text { (MC, ét. LXXXVI, 271, } 13 \text { octobre 1641) }\end{array}$ \\
\hline 1642 (10 avril) & 9000 & $\begin{array}{l}\text { seigneurie de Sainte-Colombe (MC, ét. LXXXVI, } \\
273,10 \text { avril 1642) }\end{array}$ \\
\hline 1642 (31 décembre) & 2660 & $\begin{array}{l}33 \text { a à Gisy (MC, ét. LXXV, 167, } \\
4 \text { septembre 1673) }\end{array}$ \\
\hline [1643] & {$[7000]$} & partie de la seigneurie de Sergines \\
\hline 1643 (5 février) & 11000 & $\begin{array}{l}\text { 1/10 de la seigneurie de Sergines } \\
\text { (MC, ét. LXXXVI, 275, } 5 \text { février 1643) }\end{array}$ \\
\hline 1643 (9 mars) & 54000 & $\begin{array}{l}\text { partie de la seigneurie de Sergines (MC, ét. LXXV, } \\
\text { 167, } 4 \text { septembre 1673) }\end{array}$ \\
\hline 1643 (15 mai) & 700 & $\begin{array}{l}2 \text { maisons à Michery (MC, ét. LXXV, 167, } \\
4 \text { septembre 1673) }\end{array}$ \\
\hline 1644 (21 mai) & 54000 (rente) & $\begin{array}{l}\text { terre de Gisy-les-Nobles (ADA, E* 3054, } \\
21 \text { mai 1644) }\end{array}$ \\
\hline 1644 (8 juin) & 2000 & $\begin{array}{l}\text { maison à Sergines (MC, ét. LXXV, 167, } \\
4 \text { septembre 1673) }\end{array}$ \\
\hline 1645 (24 mai) & 480 & une maison à Gisy (ADA, E* 3054, 24 mai 1645) \\
\hline 1648 (16 août) & 1400 & $\begin{array}{l}\text { moulin à Gisy (MC, ét. LXXXVI, 379, } \\
16 \text { août } 1648)\end{array}$ \\
\hline
\end{tabular}

TABLEAU 4 - Les gages, appointements et pensions

Nous pouvons nous faire une idée assez précise de ce que touche Claude Bouthillier dans l'exercice de ses fonctions grâce essentiellement à un certain nombre de documents trouvés au département des manuscrits de la Bibliothèque nationale de France et à l'ouvrage de Griselle sur la maison de Louis XIII, Marie de Médicis, Anne d'Autriche et Gaston d'Orléans. De tous les gages, appointements et pensions rassemblés dans le tableau suivant, nous ne possédons que quelques quittances ; mais de 1632 à 1643 au moins, Claude Bouthillier ayant la charge de surintendant des finances, il est probable que ce qui lui est dû est bien versé, et sans retard. 


Charge Date Montant Source

Conseiller d'État

Gages et appointements 1626

Secrétaire des comman-

dements

Gages et appointements 1619

Pension

Conseiller de la reine mère

Gages et appointements

1619

1619

$1629-1632$

$1629-1632$

Pension

Secrétaire de la chambre

Gages et appointements

Pension

Intendant des Ordres

Gages et appointements

1632

Surintendant des finances

Pension

Conseiller au Conseil de la

marine

Gages et appointements

1632

1635-1639 3000

Trésorier des Ordres

Gages et appointements

1633

1634-1652 3000

Ministre d'État

Appointements
2000

20000

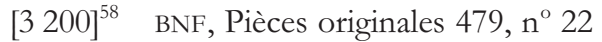

1800 E. Griselle, État de la maison de Louis XIII, Paris, 1912, p. 71

2400 Répertoire Chavigny, art. 220, fol. 20 v.

300 E. Griselle, État de la maison de Louis XIII, Paris, 1912, p. 67

BNF, Mélanges Colbert 321, fol. $29 \mathrm{v}$.

BNF, Pièces originales 479, $\mathrm{n}^{\circ} 24$; BNF, Mélanges Colbert 321, fol. $29 \mathrm{v}$.

700 E. Griselle, État de la maison de Lonis XIII, Paris, 1912, p. 28

Ibid.

Ibid.

Ibid.

Ibid.

E. Griselle, État de la maison de Louis XIII, Paris, 1912, p. 141

1800 BNF, Clairambault 1247, fol. 143 v.

20000 BNF, Mélanges Colbert 321, fol. 7 v.

2000 BNF, ms. fr. 6409, fol. 100

BNF, ms. fr. 6408, fol. 199, 255 ; ms. fr. 6409, fol. 100

BNF, Clairambault 1247, fol. $171 \mathrm{v}$. BNF, Clairambault 1247, fol. 184

BNF, Mélanges Colbert 321, fol. 3 v.

58. Cette estimation est tirée d'une quittance du $1^{\text {er }}$ décembre 1626 où sont réunis dans le même nombre les gages de conseiller d'État et de secrétaire des commandements de la reine mère. On a supposé qu'à cette date les gages pour l'office de secrétaire des commandements de la reine mère étaient toujours de 1800 livres, déduisant ainsi par soustraction les gages pour la charge de conseiller d'État. 


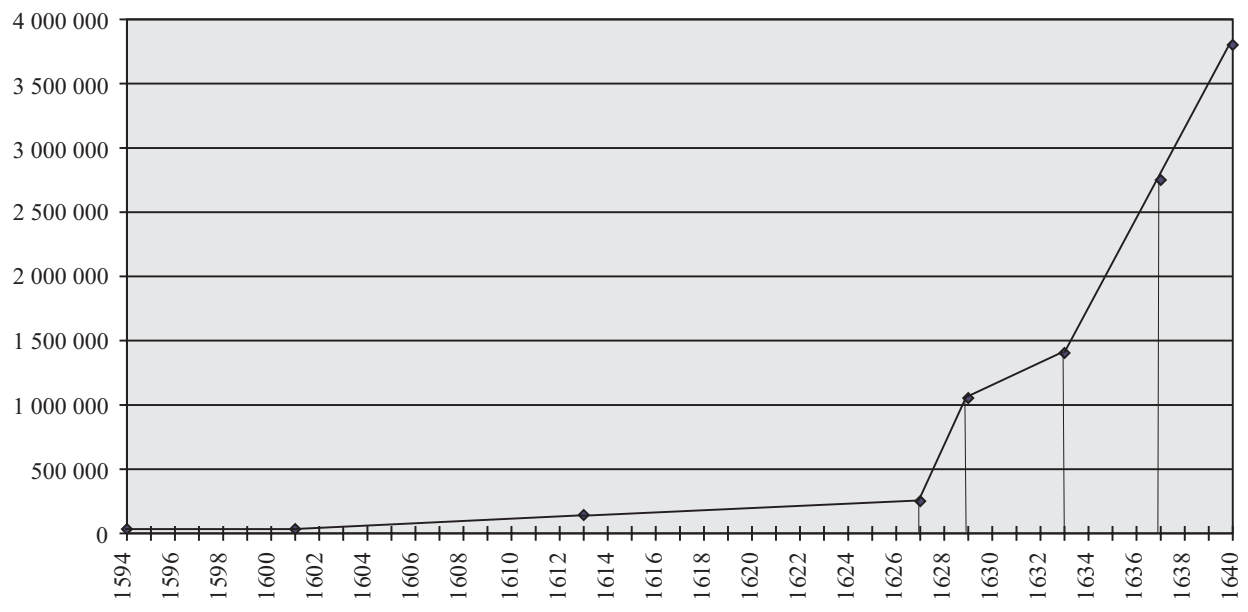

GRAPHIQUE 1 - Évolution de la fortune foncière (terres et maisons) de Claude Bullion (les sommes sont exprimées en livres)

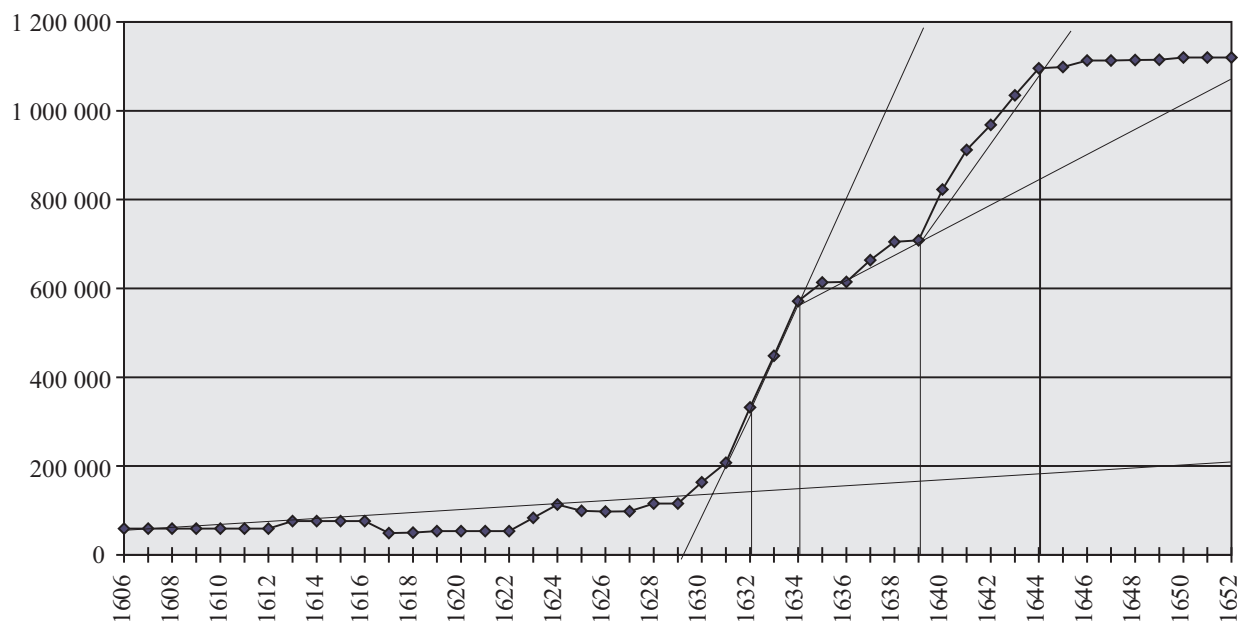

GRAPHIQUE 2 - Évolution de la fortune foncière (terres et maisons) de Claude Bouthillier (les sommes sont exprimées en livres) 


\section{TABLE DES ABRÉVIATIONS}

$\begin{array}{ll}\text { a } & \text { arpent } \\ \text { ADA } & \text { Archives départementales de l'Aube } \\ \text { AN } & \text { Archives nationales } \\ \text { bdc } & \text { boisselée de chènevière } \\ \text { BnF } & \text { Bibliothèque nationale de France } \\ \text { c } & \text { corde } \\ \text { ét. } & \text { étude } \\ \text { MC } & \text { minutier central des notaires parisiens } \\ \text { ms. fr. } & \text { manuscrit français } \\ \text { q } & \text { quartier }\end{array}$

